\title{
Nuclear transparency effect in proton and deuteron induced interactions with carbon nuclei
}

\section{Ajaz}

COMSATS Institute of Information Technology Islamabad, Pakistan

Department of Physics, Abdul Wali Khan University Mardan, Pakistan

E-mail: muhammad.ajaz@cern.ch

\section{K. Suleymanov}

COMSATS Institute of Information Technology Islamabad, Pakistan

E-mail: mais_suleymanovecomsats.edu.pk

\section{K. H. Khan}

COMSATS Institute of Information Technology Islamabad, Pakistan

E-mail: kamal,khandcern.ch

\section{A. Zaman}

COMSATS Institute of Information Technology Islamabad, Pakistan

E-mail: alizzamandcomsats.edu.pk

\section{Sh. Khalilova}

H.M. Abdullayev Institute of Physics NAS Azerbaijan Republic, Baku E-mail: Shahla.ganbarovadcern.ch

\begin{abstract}
We used the nuclear transparency effect of $\pi^{+}$-mesons in proton and deuteron induced interactions with carbon nuclei at $4.2 \mathrm{~A} \mathrm{GeV} / \mathrm{c}$ for studying the properties of nuclear matter. The average values of multiplicity, momentum and transverse momentum of the $\pi^{+}$-mesons are analyzed as a function of the number of identified protons in an event. We used the value of pseudorapidity $\left(\eta_{1 / 2}\right)$ corresponding to Half angle $\left(\theta_{1 / 2}\right)$ to see the effect of nuclear transparency for the $\pi^{+}$meson in the nuclear medium. The $\eta_{1 / 2}$ divides the multiplicity of charged particles into two equal parts in nucleon-nucleon interaction at $4.2 \mathrm{~A} \mathrm{GeV} / \mathrm{c}$. We observed several cases where the behavior could be considered as transparency effect. For quantitative description, the results are compared with cascade model. Some simple calculation using Collective Tube Model (CTM) support the idea of collective interaction with grouped nucleon encountered when propagating through the target nucleus.
\end{abstract}

XXI International Baldin Seminar on High Energy Physics Problems September 10-15, 2012

JINR, Dubna, Russia

\footnotetext{
* Speaker.
} 


\section{Introduction}

Collins and Perry [U] proposed for the first time the existence of a new phase of nuclear matter. The new phase of matter was later called the Quark-Gluon Plasma (QGP) [2] predicted by Quantum Chromodynamics [B] -the theory of strong interaction. From the last about 30 years, study of this new phase of matter get a huge attention of the experimentalist as well as theoreticians. QGP is expected to be produced at extreme conditions. It means that some critical value of temperature [四] and density is required for its production. These conditions can be produced in laboratory with the collisions of relativistic heavy nuclei at high energies. This leads to a continuing quest of leading research centers, including JINR, BNL, JLab and CERN etc, which centers on high-energy physics to create new accelerators of heavy nuclei and enhance the energies of existing accelerators. The transition of the normal nuclear matter to the new dense phase of QGP is a topic of longstanding interest in nuclear physics. A promising observable commonly used to map this transition and to identify the underlying dynamics of hadronic matter is the transparency of the nuclear medium to the propagation of hadrons. The nuclear transparency (NT) effect is connected to the changes take place in the reaction cross-section during nuclear interaction. Increase in nuclear transparency decreases the reaction cross-section. NT is defined as that an impact parameter the projectile will pass through the target nucleus with reduced interaction. It is the ratio of the cross section per nucleon to that of the cross section from a free nucleon. So, the NT provides a measure of the attenuation effects of the nuclear medium on the hadrons produced in a reaction. Energy dependence of the NT

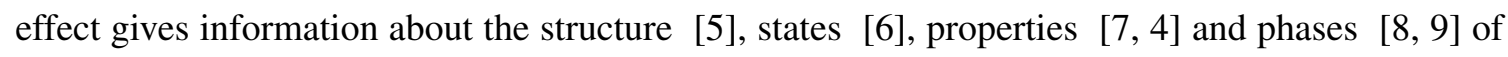
nuclear matter. The idea of nuclear transparency in collisions was apparently first introduced by $\mathrm{H}$. A. Bethe [ए]] in 1940. An anomalous increase in the behavior of NT is the phenomenon of color transparency (CT) predicted by quantum chromodynamics (QCD). The concept of CT was first introduced by Brodsky and Mueller [], [2] in 1982. CT is the prediction reduced interactions [[ए3] of hadrons produced in exclusive reactions with high four momentum transfers squared $\left(Q^{2}\right)$ (with $\mathrm{r}_{\perp} \sim\left(\frac{1}{Q}\right)$. A lot of efforts have been made to search for the CT effect. Results of quasi-elastic A(p,

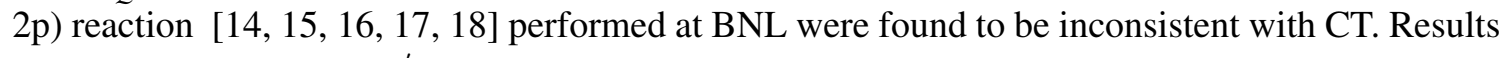
of the quasi-elastic A(e, e' p) reaction [ㅁ, 201, 21], 22, 22], 24] at SLAC with the A-dependence as a function of $Q^{2}$ also did not produce the effect of CT. In later studies mesons were used with the premise that an earlier onset of CT for mesons production is expected than that for baryons because the at large $Q^{2}$ the values of $\mathrm{r}_{\perp} \sim\left(\frac{1}{Q}\right)$ is significantly smaller than that for nucleons. The results include $\rho$-meson production [25, [26] [Hermes Collaboration ], diffractive dissociation of pions into di-jets [27] and pion photoproduction process [28, [29] at JLab. The intrested reader for a more complete review on the nuclear transparency is referred to a status report by Dutta et al [30]].

\section{The Method and Experiment}

Using the idea of the half angle method [ [6] we used the value of pseudorapidity $(\eta)$ which divides the multiplicity of all charged particles into two equal parts denoted by $\eta_{1 / 2}$. Using pp collision we determined the values of the $\eta_{1 / 2}$ to be 1.51 . The particles with $\eta>\eta_{1 / 2}$ are the incone particles and those with $\eta<\eta_{1 / 2}$ are the outcone particles. We defined the NT as an effect at which the characteristics of $\pi^{+}$-mesons in proton induced interactions with carbon nuclei (pC) 
and deuteron induced interactions with carbon nuclei (dC) do not depend on $N_{p} . N_{p}$ is used to fix the baryon density of nuclear collisions. Besides $\eta_{1 / 2}$, we used five different values of $\eta$ including $1.74,2.03,2.44$ and 3.13 corresponding to $\theta=20^{\circ}, 15^{\circ}, 10^{\circ}$, and $05^{\circ}$ respectively to check the behavior of the average characteristics at these different values. Finally the results are compared with the data coming from Dubna version of cascade model [ [3], 132, [34, [3]]. The cascade model is a popular model, used to describe the general features of relativistic nucleus-nucleus collisions. It is an approach based on simulation using Monte-Carlo techniques and is applied to situation where multiple scattering is important. The basic assumptions and procedures of the cascade model are given by K. K. Gudima et al. [B]] and A. Boudard et. al [B]]. We also used some simple calculation using expressions from the Collective Tube Model (CTM) [B3]]. In the CTM it is assumed that the incoming particle interacts with the tube that lies along its path. It means that the particle/nucleons in the target that lies in the path of the incident particle interact collectively to the latter. In the independent particle approximation for the nucleus, the probabilities $P(i, A)$ for the projectile to encounter a tube with $i$ nucleons is calculated with the assumption that the tube act as a big hadron. We used the experimental data obtained from the 2-m propane $\left(\mathrm{C}_{3} \mathrm{H}_{8}\right)$ bubble chamber of the High Energy Laboratory (LHE) of the Joint Institute for Nuclear Research (JINR), Dubna, Russia. The chamber was exposed to beams of protons and deuterons accelerated to a momentum of $4.2 \mathrm{~A} \mathrm{GeV} / \mathrm{c}$. A magnetic field of 1.5 tesla was used to separate the positively and negatively charged particle, as well as to calculate the momentum of the high energy particles from their trajectories. Threshold values for the momentum charged pions was set to $70 \mathrm{MeV} / \mathrm{c}$, whereas the threshold for protons momentum was set to $150 \mathrm{MeV} / \mathrm{c}$ respectively below which the respective hadrons were not identified because of their short range in the chamber. Protons was identified upto $500 \mathrm{MeV} / \mathrm{c}$ with certainity beyond which there was a difficulty in the identification of the protons with the $\pi^{+}$-mesons. All positive charged particles above $750 \mathrm{Mev} / \mathrm{c}$ were considered as protons. All negative charged particles (expect identified electrons) were considered as $\pi^{-}$-mesons. Secondaries having $p>3 \mathrm{GeV} / \mathrm{c}$ and $\theta<4^{\circ}$ are stripping fragments while the proton participants are those whose $p>300 \mathrm{MeV} / \mathrm{c}$ excluding the stripping fragments. An average error in angles was measured to be $0.8^{\circ}$, whereas the average relative error in momenta of the secondary particles from the curvature of their track in the magnetic field was $11 \%$. The detail discussion on the interaction mechanism is given by H. N. Agakishiyev et al. [B6]. In this experiment, we used $12757 \mathrm{pC}$ - and $9016 \mathrm{dC}$ - interactions at a momentum of $4.2 \mathrm{~A} \mathrm{GeV/c}$. In the case of cascade code we used 50000 $\mathrm{pC}-$ and $\mathrm{dC}$-interactions under the same conditions.

\section{Results}

The average multiplicity $<\mathrm{n}>$ of the outcone $\pi^{+}$-mesons in $\mathrm{pC}$ - interactions at $\eta_{1 / 2}=1.51$ as well as for $\eta=1.74,2.03,2.44$ and 3.13 as a function of the $N_{p}$ are shown in Fig.1(a). Experimental data results are shown by geometrical symbols while the results of the cascade model are shown by lines as given in legend against each value of $\eta$. The results from the $\mathrm{dC}$ data under the same conditions are given in Fig. 1(b) having the same representation of geometrical symbols and lines for experimental data as well as cascade model respectively. The values of $<\mathrm{n}>$ in experimental data of pC- and dC- interactions decreases sharply with $N_{p}=0$ - 2 and then increases slowly, but the results in the case of Cascade model is unable to reproduced the results completely. In the case 
of $\mathrm{pC}$ cascade model can qualitatively describe the results but not in the case of $\mathrm{dC}$ data. We did not observe any signal on transparency in the outcone $<n>$ of $\pi^{+}$-mesons. The $<n>$ is suppressed in experimental data as compared to cascade model. The reason being the possibility of mixing of some of the fast $\pi^{+}$-mesons with protons as is explained in [37]. The values for the $<\mathrm{p}>$ as a function of the $N_{p}$ for these pions in $\mathrm{pC}$ and $\mathrm{dC}$ data are givem in Fig. 1(c) and 1(d) respectively for different values of $\eta$. The results of the experimental and the code data are substantially different. The experimental data shows transparency for almost all values of $\eta$ including $\eta_{1 / 2}$, whereas the $<\mathrm{p}>$ decreases linearly with increasing $N_{p}$ for all values of $\eta$. It means that the observed transparency is not reproduced by the cascade model. Fig. 2(e) and 2(f) demonstrate $\left\langle\mathrm{p}_{T}\right\rangle$ as a function of the $N_{p}$ for the $\mathrm{pC}$ and $\mathrm{dC}$ data respectively. One can see a clear differences between experimental and code data. Experimental data shows transparency with some small degree of oscillation. The code data could not explain the results of experimental data and show a decreasing behavior of $\left\langle p_{T}\right\rangle$ with increasing $N_{p}$. So we could say that the experimental data on behavior of the average characteristics of the out cone $\pi^{+}$-mesons demonstrate some transparency which could not be described by the cascade model. This behavior could not be the reason of leading effect due to the fact that the outcone $\pi^{+}$-mesons are secondary produced particles having small energy and $\eta$ less than $1.51,1.74$, 2.03, 2.44 and 3.13. In the $\mathrm{pC}$ - and $\mathrm{dC}$ - interactions transparency seems to be more clean on level of about $\left\langle p_{T}\right\rangle \approx 0.26 \mathrm{GeV} / \mathrm{c}$. We can roughly calculate the length of the tube $(R)$ radiating pions through the poins $\left\langle p_{T}\right\rangle$ as $\mathrm{R} \sim \frac{1}{\left.<p_{T}\right\rangle}$. Using the approximate expression the value of $R \sim 3.8 \mathrm{fm}$ . Diameter of the carbon nucleus is approximately equal to 3.67 which is the same as that of the length of the tube radiating pion in the out cone pions. As we could see the projectile didnt change almost the values of the $R$ because in $\mathrm{pC}$ - and $\mathrm{dC}$ - interactions the values of $\left\langle p_{T}\right\rangle \sim 0.26 \mathrm{GeV} / \mathrm{c}$. So we can say that a reason of the observed transparency is that the size of pion radiation area doesnt change during interaction with increase in the number of protons as well as with the mass of the projectile. The transparency observed for the outcone $\pi^{+}$-mesons is not reproduced by the cascade model. It means that the behavior is connected to some effect which is beyond the scope of the model. Y. Afek et, al., [39] has broadly divided the various models that have been suggested so far for high energy particle-nucleus collisions and for high energy nucleus-nucleus collisions into two categories. The first category of models includes those models which assume that particle nucleus collision is a single step process consisting of successive independent collisions with nucleon in the nucleus while propagating through it. Cascade model is a typical example of the first category of models. The center of mass energy available for the production of new particles in these models is given by $s \lesssim 2 m P_{l a b}$. The second category included all those models which assume that particle-nucleus collisions in a single step process where a few nucleons in the nucleus respond collectively to the incident particle. Collective Tube Model (CTM) [39, 40] is a typical example of the second category of models. In such models the effective center of mass energy available for the production of particles is approximately given by $s_{e f f} \simeq 2 m_{e f f} P_{l a b}$. Where $m_{e f f}$ is the mass of the system that interacts collectively to the incident particle. The value of this mass is of the order of a few times the mass of a single nucleon. $P_{l a b}$ is a momentum of the incident particles. The model has been successfully applied to various processes at low and high $p_{T}$ [41, 42, 43, 44, 45]. We think that the observed transparency could be considered as a result of the collective interaction of grouped nucleons with the proton and deuteron in $\mathrm{pC}$ - and $\mathrm{dC}$ - interactions. 
(a)

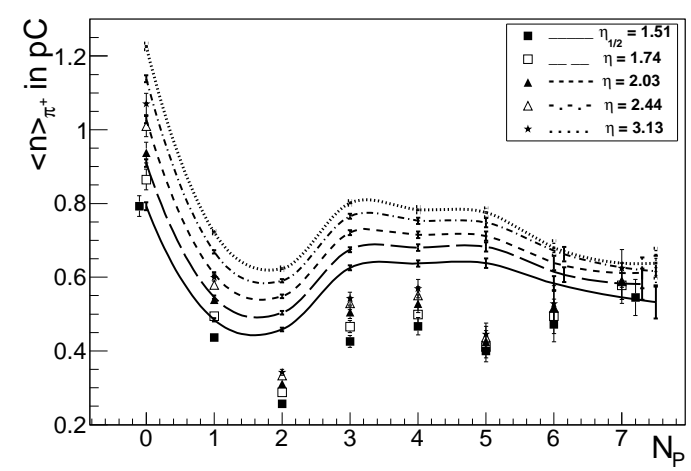

(c)

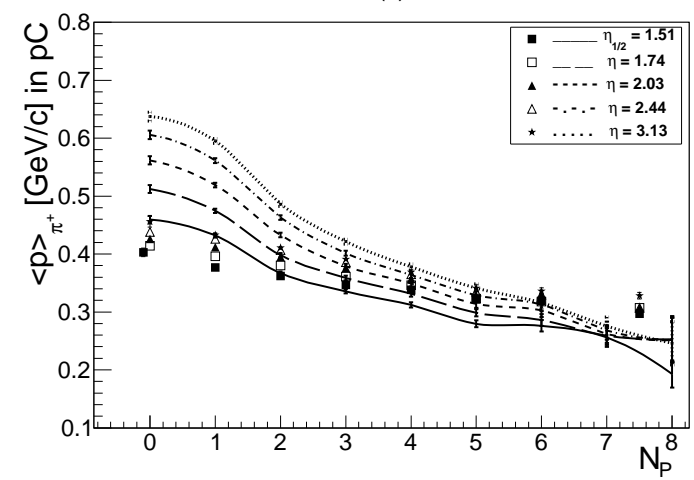

(e)

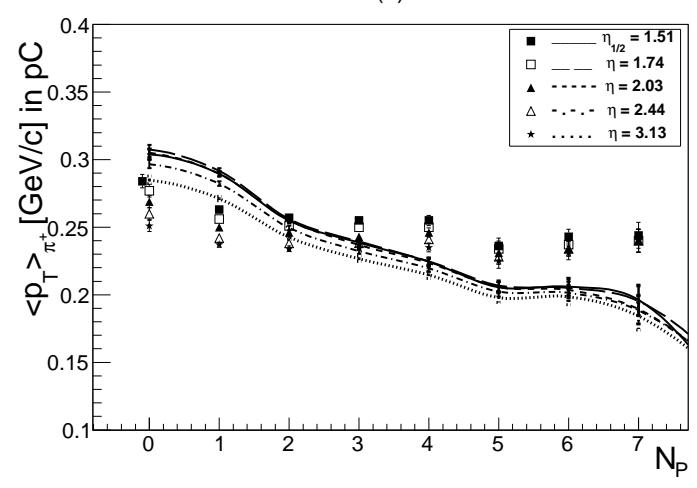

(b)

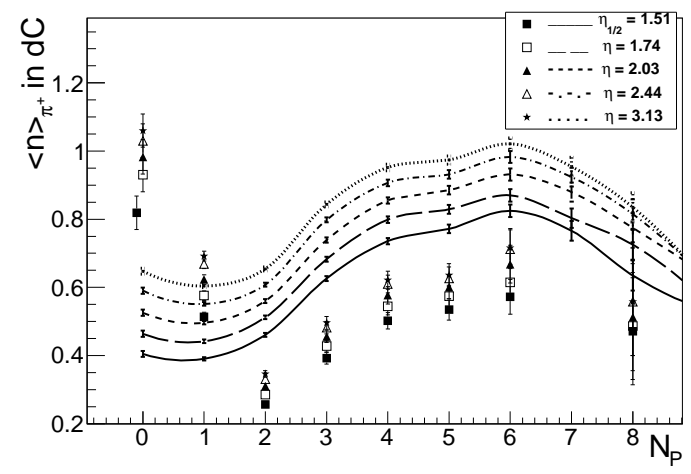

(d)

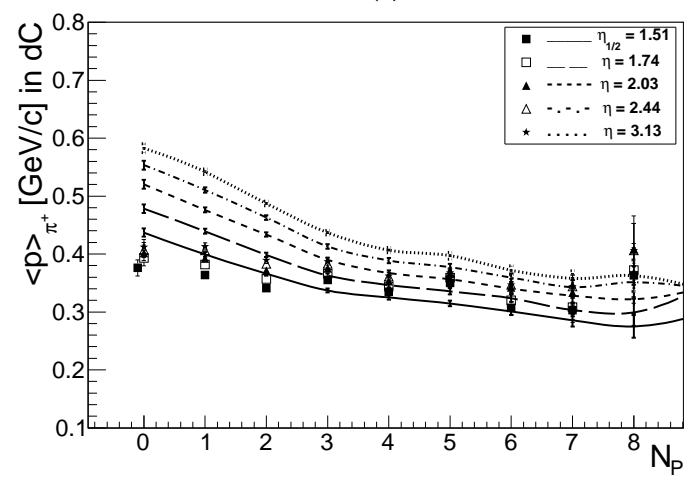

(f)

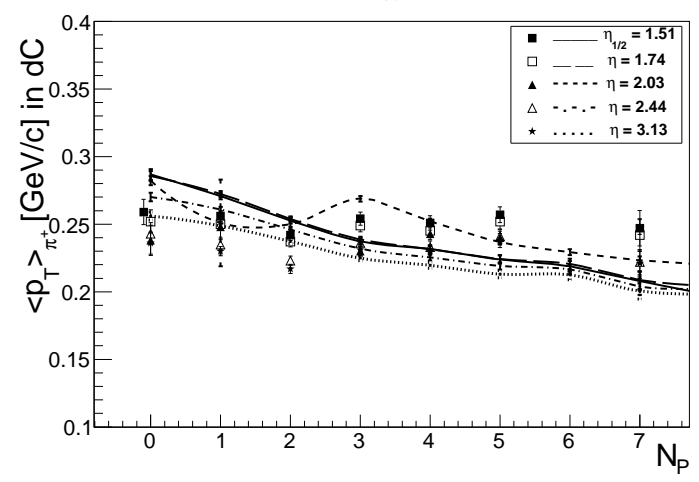

Figure 1: Behavior of the average characteristics of outcone $\pi^{+}$-mesons as a function of number of identified protons $\left(N_{p}\right)$. Fig 1(a) is the behavior of average multiplicity $(<n>)$ of outcone $\pi^{+}$-mesons as a function of $N_{p}$ in pC-interactions, 1 (b) is the $\left\langle n>\right.$ of outcone $\pi^{+}$-mesons as a function of $N_{p}$ in dC- interactions, 1 (c) is the $\langle p\rangle$ versus $N_{p}$ in $\mathrm{pC}$ - and $1(\mathrm{~d})$ is $\langle p\rangle$ in dC- interactions, $1(\mathrm{e})$ is the $\left\langle p_{T}\right\rangle$ versus $N_{p}$ in pCand 1 (f) is the $\left\langle p_{T}\right\rangle$ in $\mathrm{dC}$ - interactions. 
Using the following expression from CTM [B] ] for the average multiplicity of secondary charged particles $(<\mathrm{n}(\mathrm{s})>)$ as a function of the number of fast protons $N_{p}$, we can calculate roughly the number of nucleons in tube $\left(i_{p}\right)$.

$$
<n(s)>_{i p} \simeq<n(s)>_{p p} \mathrm{x}\left(<i_{p}>\right)^{\alpha}
$$

here $\left\langle n(s)>_{i p}\right.$ is the multiplicity of the secondary charged particles produced in tube with ip nucleons whereas $\left\langle n(s)>_{p p}\right.$ is multiplicity of the secondary charged particles in pp-interaction at same energy in center of mass. $\mathrm{A}$ and $\mathrm{Z}$ are the mass number and charge number of target nucleus respectively (for carbon nucleus $\mathrm{A}=12, \mathrm{Z}=6$ ). For estimation we take $\alpha \sim \frac{1}{4}$ [ [46] so we get $i_{p} \sim 3.1 \pm 0.3$ (the average values) which is almost independent of the $\eta$ The diameter of the carbon nucleus is about $3.6 \mathrm{fm}$ which can accommodate 2.5 nucleons in its diameter. Our calculated value for the number of nucleons in the tube is close to the number of nucleon in the diameter of the carbon nucleus. This result give us opportunity to say that the collective interaction of grouped nucleons in the nucleus / nuclear medium is a reason for observed transparency for out cone $\pi^{+}$-mesons produced in $\mathrm{pC}$ - and $\mathrm{dC}$-interactions.

\section{Summary and Conclusion}

For looking the properties of nuclear matter, we used the nuclear transparency effect of the $\pi^{+}$-mesons. To get a signal on the NT, we studied the average characteristics of $\pi^{+}$-mesons using $\mathrm{pC}$ - and $\mathrm{dC}$ - interactions at $4.2 \mathrm{~A} \mathrm{GeV/c}$. Using the idea of half angle technique we used the value of $\eta$ which divides the charged particles multiplicty into two parts. We observed some signal of the nuclear transparency effect in the behavior of outcone $\left(\eta_{1 / 2} \leq 1.51\right.$ as well as for $\eta \leq$ $1.74,2.03,2.44$ and 3.13) $\pi^{+}$-mesons. We consider the effect of nuclear transparency as some signal which could be connected to some particular properties of the medium. The results of the experimental data are compared with the cascade model and Coherent Tube Model. Cascade model could not explain the results which were used as a typical example of the first category of models. The transparency appears due to the collective interaction of the grouped nucleon which interact collectively with the incident particle. The number of nucleon which responds collectively to the interaction of incident particle is calculated using CTM which comes out to be about 3 . We have also calculated the length of the tube radiating pions which come out to be the same as that of the diameter of the carbon nucleus. So we could conclude that the source of these particles could be the collective interaction of target nucleons with the projectile.

\section{References}

[1] J. C. Collins, M. J. Perry, "Superdense Matter: Neutrons or Asymptotically Free Quarks?", Phys. Rev. Lett. 34, (1975) 1353.

[2] S. Jeon, V. Koch, "Review for Quark-Gluon Plasma" 3. eds. Hwa R C and Wang X-N, Singapore: World Scientific, (2004).

[3] W. Greiner and A. Schafer, "Quantum Chromodynamics". Springer, (1994). 
[4] M. Ajaz, M. K. Suleymanov, K. H. Khan and A. Zaman, "Searching for the properties of nuclear matter using proton-carbon and deuteron-carbon collisions at $4.2 \mathrm{~A} \mathrm{GeV/c"} \mathrm{Int.J.Mod.Phys.} \mathrm{E,} \mathrm{21,}$ (2012) 1250095.

[5] S. Szilner, F. Haas and Z. Basrak, "Weak absorption and resonances in light heavy ion reactions induced by the non- $\alpha$-type ${ }^{14}$ C nucleus", FIZIKA B 12, 2, (2003) 117.

[6] A. I. Anoshin et al. "Investigation of $\pi^{-}$-mesons with carbon nucleus at $P \pi^{-}=40 \mathrm{GeV} / \mathrm{c}$ and the effect of "Nuclear Transparency" for high energy hadrons", Sov. Journal of Nucl. Phys., 27, (1978) 1240.

[7] H. Satz. "Deconfinement and percolation.", Nucl. Phys. A, 642, (1998), 130.

[8] W. Cosyn et al., "Color transparency and short-range correlations in exclusive pion photo- and electroproduction from nuclei.", Phys. Rev., C 77, (2008), 034602

[9] M. Ajaz, M. K. Suleymanov, K. H. Khan and A. Zaman, "Study of the behavior of the nuclear modificatioin factor in freeze-out state", Chin.Phys. C (HEP\&NP), 37, (2013) 024101.

[10] H. A. Bethe, "A Continuum Theory of the Compund Nucleus", Phys. Rev. 57, (1940) 1125.

[11] S. J. Brodsky, "Factorization and other Novel Effects in QCD", Proceeding of the XIII International Symposium on Multiparticle Dynamics, Volendam, Netherlands, eds, E. W. Kittel, W. Metzger and A. Stergion "Worls Scientific, Singapor" (1982) 963.

[12] A. H. Mueller, Proceedins of the XVII rencontre de Moroind, Les Arcs, France, ed. Tran Than Van (Editions Frontieres, Gif-sur-Yvette), 1, (1982) 13.

[13] S. J. Brodsky and A. H. Mueller, "Using nuclei to probe hadronization in QCD", Phys. Lett. B 206 (1988) 685.

[14] A. S. Carroll et al., "Nuclear Transparency to Large-Angle pp Elastic Scattering", Phys. Rev. Lett. 61, (1988) 1698.

[15] I. Mardor et al., "Effect of multiple scattering on the measurement of nuclear transparency", Phys. Rev. C, 46, (1992) 761.

[16] I. Mardor et al., "Nuclear Transparency in Large Momentum Transfer Quasielastic Scattering", Phys. Rev. Lett. 81, (1998) 5085.

[17] A. Leksanov et al., "Energy Dependence of Nuclear Transparency in ${ }^{12} C(p, 2 p)$ Scattering", Phys. Rev. Lett. 87, (2001) 212301.

[18] J. L. S. Aclander et al., "Nuclear transparency in $90_{c . m}^{o}$ quasielastic A(p,2p) reactions", Phys. Rev. C 70, (2004) 015208.

[19] G. Garino et al., "Proton propagation in nuclei studied in the (e, é p) reaction", Phys. Rev. C 45, (1992) 780 .

[20] N. C. R. Makins et al., "Momentum Transfer Dependence of Nuclear Transparency from the Quasielastic ${ }^{12}$ C(e, é p) Reaction", Phys. Rev. Lett. 72, (1994) 1986.

[21] T. G. ONeill et al., "A-dependence of nuclear transparency in quasielastic A(e, é p) at high $Q^{2}$ ", Phys. Lett. B 351, (1995) 87.

[22] D. J. Abbott et al., "Quasifree (e,ép) reactions and proton propagation in nuclei,", Phys. Rev. Lett. 80, (1998) 5072.

[23] K. Garrow et al., "Nuclear transparency from quasielastic $A\left(e, e^{\prime} p\right)$ reactions up to $Q^{2}=8.1$ $(\mathrm{GeV} / \mathrm{c})^{2}$ ", Phys. Rev. C 66, (2002) 044613. 
[24] D. Dutta et al. (Jefferson Lab E91013), "A Study of the Quasi-elastic (e é p) Reaction on ${ }^{12} C,{ }^{56} \mathrm{Fe}$, and ${ }^{97} A u^{\prime \prime}$, Phys. Rev. C 68, (2003) 064603.

[25] M. R. Adams et al. (E665), Measurement of Nuclear Transparencies from Exclusive $\rho^{\circ}$ Meson Production in Muon-Nucleus Scattering at 470 GeV, Phys. Rev. Lett. 74, (1995) 1525.

[26] A. Airapetian et al. (HERMES), "The $Q^{2}$ dependence of nuclear transparency for exclusive $\rho^{o}$ production", Phys. Rev. Lett. 90, (2003) 052501.

[27] E. M. Aitala et al. (E791), "Observation of color-transparency in diffractive dissociation of pions", Phys. Rev. Lett. 86, (2001) 4773.

[28] D. Dutta et al. (Jefferson Lab E940104), "Nuclear transparency with the $\gamma n \rightarrow \pi^{-}$p process in ${ }^{4} H{ }^{\prime \prime}$, Phys. Rev. C 68, (2003) 021001(R).

[29] B. Clasie et al., "Measurement of Nuclear Transparency for the A(e, é $\left.\pi^{+}\right)$Reaction", Phys. Rev. Lett. 99, (2007) 242502.

[30] D. Dutta and K. Hafidi, " The search for the onset state of color transparency: A status report", Int. J. Mod. Phys. E 21, (2012), 1230004.

[31] K. K.Gudima, V. D. Toneev., "Particle emission in light and heavy ion reactions", Nucl. Phys. A 400 (1983) 173.

[32] A. Boudard et. al, "Intranuclear cascade model for a comprehensive description of spallation reaction data", Phys. Rev. C 66 (2002) 044615.

[33] Y. Afek, G. Berlad, A. Dar and G. Eilam, "The Collective Tube Model for high energy particle-nucleus and nucleus-nucleus collisions", THC11NI0N - PH - 76 - 87 (1976).

[34] V. S Barashenkov et al., "Intranuclear Cascade Studies Of Inelastic Nucleus-nucleus Interactions.". Sov.J.Nucl.Phys. 39, (1984) 715, Yad.Fiz. 39, (1984) 1133, JINR Preprint P2-83-117 (Dubna, 1983).

[35] M. I. Adamovich, et. al., "FRAGMENTATION AND MULTIFRAGMENTATION OF 10.6A GeV GOLD NUCLEI", Z. Phys. A 358 (1997)

[36] A.I. Bondarenko et. al., "The Ensemble of interactions on carbon and hydrogen nuclei obtained using the $2 \mathrm{~m}$ propane bubble chamber exposed to the beams of protons and $\mathrm{H}^{2}, \mathrm{He}^{4}, \mathrm{C}^{12}$ relativistic nuclei at the Dubna Synchrophasotron. ", JINR Communications P1-98-292, Dubna (1998)

[37] M. Ajaz, M. K. Suleymanov, K. H. Khan and A. Zaman, "Searching for the properties of nuclear matter using proton-carbon and deuteron-carbon collisions at $4.2 \mathrm{~A} \mathrm{GeV/c.} \mathrm{"} \mathrm{Int.J.Mod.Phys.} \mathrm{E,} \mathrm{21,}$ 12 (2012) 1250095 and references therein

[38] S. Karataglidis, A. I. Wright, "Charge exchange reactions as tests for structures of exotic nuclei" 12th International Conference on Nuclear Reaction Mechanisms, Villa Monastero, Varenna, Italy. (2009) 115 .

[39] Y. Afek, G. Berlad, A. Dar and G. Eilam, "The Collective Tube Model for high energy particle-nucleus and nucleus-nucleus collisions", THC11NI0N - PH - 76 - 87 (1976).

[40] G.Berlad, A. Dar and G. Eilam, "Multiparticle production in particle nucleus collisions at high energies", Phys. Rev. D, 13, 161 (1976).

[41] Y. Afek, G. Berlad, A. Dar and G. Eilam, "Scaling laws for inclusive production of hadrons in high-energy particle-nucleus collisions", Phys. Rev. D, 15, 2622 (1978)

[42] Y. Afek, G. Berlad, A. Dar and G. Eilam, "Erratum: Scaling laws for inclusive production of hadrons in high-energy particle-nucleus collisions", Phys. Rev. D, 17, 376(E) (1978) 
[43] S. Fredriksson, Nucl. Phys.B, "Recent results from the Tube Model for high-energy collisions with nuclei", 111, 167 (1976)

[44] Y. Afek, G. Berlad, A. Dar and G. Eilam, "Multi-pion Production in Relativistic Heavy Ion Collisions" Phys. Rev. Lett., 41, 849 (1978)

[45] Y. Afek, G. Berlad and G. Eilam, "Production of W bosons in proton-nucleus collisions", Phys. Rev. D, 20, 1160, (1979)

[46] N.A. Kobylinsky, E.S. Martinov, V.P. Shelest, "Hadronic multiplicity and total cross-section: A new scaling in wide energy range" Z.Phys.C-Particles and Fields 28 (1985) 143. 